\title{
HI observations of nearby southern Seyfert galaxies with the ATNF/Mopra radiotelescope
}

\author{
Alcides G. R. Adornes ${ }^{1}$, Rosane Bohrer-Adornes ${ }^{1}$, \\ Everton Lüdke ${ }^{1}$ and Thaisa Storchi-Bergmann ${ }^{2}$ \\ ${ }^{1}$ Universidade Federal de Santa Maria, CCNE - Departamento de Física - LARIE, Campus \\ Universitário, Santa Maria RS 97150-900, Brazil \\ ${ }^{2}$ UFRGS, Instituto de Física, Departamento de Astronomia, Porto Alegre RS 91501-970, Brazil
}

\begin{abstract}
We present single-dish observations of a sample of 27 nearby $(z<0.018)$ Seyfert galaxies obtained with the ATNF/Mopra radiotelescope. The total neutral gas content for the sample is derived. We find that Seyfert galaxies are gas-rich objects with a median HI mass to blue luminosity ratio $M_{H I} / L_{B} \sim 0.38 \mathrm{M}_{\odot} / L_{\odot}$, which tends to increase with decreasing optical and far-infrared luminosities. We have also found a correlation among the neutral hydrogen content of Seyfert 2 galaxies and the IRAS luminosities, similar to those found for normal spiral and starburst galaxies. Our results suggest a scenario where the star formation of Seyfert galaxies is similar to that found in starbursts, and that they are dependent of the initial neutral mass content of progenitors of galaxy disks in the blue compact-HII galaxy-Seyfert sequence. Within this sequence, the far-infrared luminosity and the total hydrogen mass correlate as $L_{f i r} / L_{\odot} \propto\left(M_{H I} / M_{\odot}\right)^{1.5 \pm 0.4}$.
\end{abstract}

\section{Introduction}

In Seyfert galaxies, the nuclear activity and the starburst phenomenon need "fuel", and may be associated with mass transfer from the outer parts of the host galaxy. One possible mechanism is mass transfer phenomena in galaxy pairs. It is believed that dynamical interactions may force cold gas into the nuclear regions and provide the "fuel" needed to feed the central engine in the Seyfert nucleus or to trigger violent star forming episodes, which result in blue luminous starbursts.

We therefore have made an HI spectral line survey of nearby seyfert galaxies with the Mopra radiotelescope to study the molecular and optical/infrared continuum emission. We also compared the data with published samples of compact dwarf galaxies and starburst galaxies to look for observational trends, which are summarized in this paper.

\section{Conclusions}

Using the Mopra radiotelescope, we have provided total hydrogen masses for a sample of southern seyfert galaxies (declination $<-10^{\circ}$ ), selected from standard catalogues of AGNs, up to the redshift of $z \sim 0.018$.

A comparison with sub-samples of published HI surveys shows that the blue luminosities, magnitudes and the far-infrared luminosities correlate well with the total neutral hydrogen content (figure 1), suggesting that differences in seyferts, starbursts and blue compact galaxies can be explained by star formation, which is dependent on the total gas content available in each individual galaxy during its past history. In fact, the most 


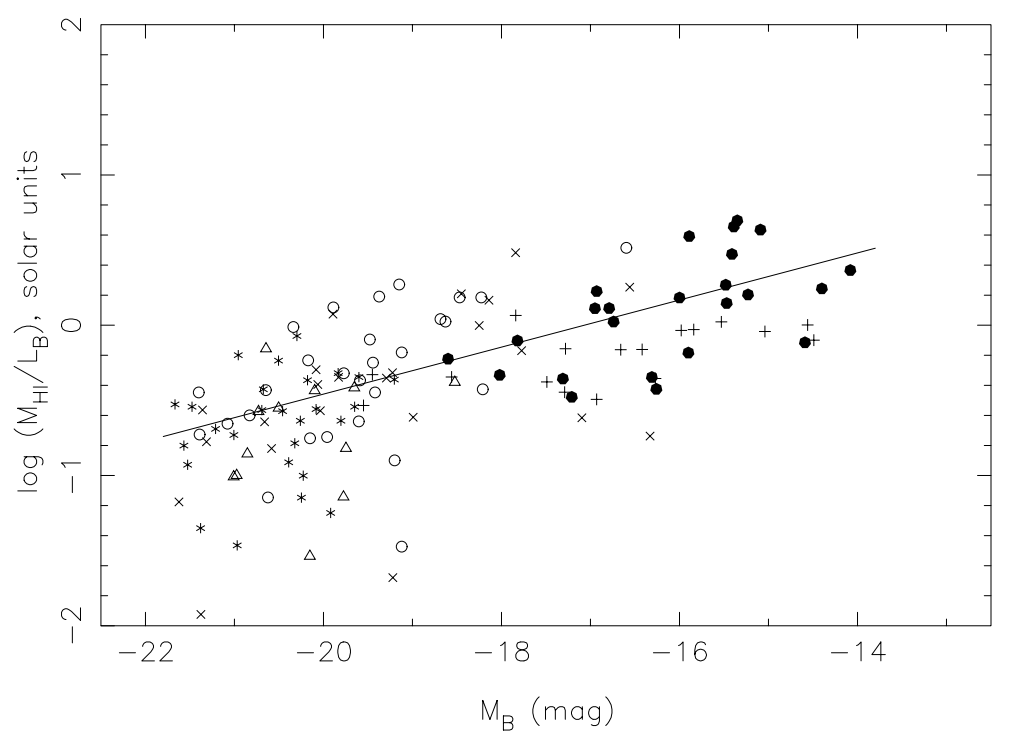

Figure 1. HI mass plotted against blue magnitudes. Our sample is plotted with open circles. Data from blue compact dwarfs (plus) and gas-rich irregular galaxies (filled circles) from Smoker et al. (2000) tables 3 and 4 are also plotted. Open triangles are more distant seyfert galaxies $\left(2900<V_{r}<7800 \mathrm{~km} \mathrm{~s}^{-1}\right)$ observed by Blank (2000) with the ATNF Parkes 64-m telescope. A sample of flattened galaxies with outer gas rings observed with the late 100-foot Green Bank telescope by Richter et al. (1994) is also included (crosses). Northern seyfert galaxies observed with the Arecibo Observatory by Mirabel \& Wilson (1984) are plotted with $*$ symbols.

luminous starbursts and the weakest Seyfert galaxies appear to overlap in global optical properties, having similar total HI content.

The history of star formation also predicts that at a given epoch, part of the neutral gas is converted to stars. The far-infrared emission is certainly due to radiation arising from dusty HII, and giant HII regions and the direct heating of dust by stellar populations. Devereux \& Young (1991) have discussed this possibility in an analysis of far-infrared luminosities of a large population of normal spiral galaxies, and that a trend between $L_{i r}$ and $\mathrm{H}_{\alpha}$ luminosities show a tight correlation. Seyfert galaxies and gas-rich starbursts show a correlation between $L_{f i r}$ and the neutral hydrogen content which gives additional support to the idea that Seyfert galaxies have undergone stronger star formation than dwarf galaxies, and the scatter in the plots may be due to dispersions in the distribution of stellar populations of hot globular clusters in present-day galaxies. It is also worth mentioning that the morphological types do overlap in the correlations, meaning that for a given $M_{H I}$, starbursts and Seyferts may have similar luminosities in the far-infrared and in the blue band.

\section{Acknowledgements}

The ATNF/CSIRO is funded by the British Commonwealth.

\section{References}

Blank, D. L. 2000, PhD Thesis, School of Physics, University of Sydney, Sydney/NSW, Australia Devereux, N. A., \& Young, J. S. 1991 ApJ, 371, 515

Mirabel, I. F., \& Wilson, A. S. 1984, ApJ, 277, 92

Richter, O.-G., Sackett, P. D., \& Sparke, L. S. 1994, AJ, 107, 99

Smoker, J. V., Davis, R. D., Axon, D. J., \& Hummel, E. 2000, A\&A, 361, 19 